\title{
Diagnostic evaluation of rapid tests for scrub typhus in the Indian population is needed
}

\author{
Siddharudha Shivalli
}

\begin{abstract}
Background: Owing to frequent outbreaks witnessed in different parts of the country in the recent past, scrub typhus is being described as a re-emerging infectious disease in India. Differentiating scrub typhus from other endemic diseases like malaria, leptospirosis, dengue fever, typhoid, etc. is difficult due to overlapping clinical features and a lower positivity for eschars in Asian populations. Hence, the diagnosis heavily relies on laboratory tests.

Discussion: Costs and the need of technical expertise limit the wide use of indirect immunoperoxidase or immunofluorescence assays, ELISA and PCR. The Weil-Felix test is the most commonly used and least expensive serological test, but lacks both sensitivity and specificity. Hence, the diagnosis of scrub typhus is often delayed or overlooked. With due consideration of the cost, rapidity, single test result and simplicity of interpretation, rapid diagnostic tests have come into vogue. However, evaluation of rapid diagnostic tests for scrub typhus in the Indian population is needed to justify or discourage their use.

Conclusion: Research studies are needed to find the most suitable test in terms of the rapidity of the result, simplicity of the procedure, ease of interpretation and cost to be used in the Indian populace.
\end{abstract}

Keywords: India, Rapid test, Scrub typhus

\section{Multilingual abstract}

Please see Additional file 1 for translations of the abstract into the six official working languages of the United Nations.

\section{Introduction}

Scrub typhus is caused by Orientia tsutsugamushi and is transmitted by the bite of infected larvae of the mite Leptotrombidium deliense. It is a zoonosis, with humans being accidental, dead end hosts. India is an integral component of the "tsutsugamushi triangle" which depicts a part of the globe (northern Japan and eastern Russia in the north, northern Australia in the south, and Pakistan in the west) endemic to scrub typhus. Owing to frequent outbreaks witnessed in different parts of the country in the recent past, it is being described as a reemerging infectious disease in India. It is one of the

Correspondence: shivalli.bhu@gmail.com

Department of Community Medicine, Yenepoya Medical College, Yenepoya University, Mangalore-575018 Karnataka, India most under-diagnosed and under-reported febrile illnesses requiring hospitalization in the region. Eschar (painless, punched out ulcer up to $1 \mathrm{~cm}$ in width with a black necrotic centre) is an important finding for the diagnosis of scrub typhus. However, eschar positivity in India and other Asian populations is very low [1]. The absence of an eschar does not rule out scrub typhus in Indian context and hence, the diagnosis relies heavily on laboratory tests.

\section{Discussion}

Scrub typhus is essentially an occupational disease among the rural residents (i.e. farmers, those involved in collecting firewood from the jungle, fishing in the pond etc.) in the Asia-Pacific region [2]. Many tests are available for the diagnosis of scrub typhus and each test has its own advantages and limitations. Indirect immunoperoxidase and immunofluorescence assays are the gold standards for diagnosis [3]. However, the cost and need of technical expertise limit their wide use. Similarly, Enzyme Linked Immunosorbent assay (ELISA) and Ploymerase 
Chain Reaction (PCR) are not available beyond the secondary level of health care like district hospitals in India [3]. ELISA is easy and relatively economical, but samples need to be pooled, which may delay the diagnosis and affect the overall disease outcome. Many of these tests are not available in remote/rural areas where most cases occur [2]. Weil-Felix test is a commonly used in-expensive serological test that lacks both sensitivity and specificity [4].

Differentiating scrub typhus from other endemic diseases like malaria, leptospirosis, dengue fever, and typhoid is often difficult for a clinician. Lower positivity for an eschar in Asian populations and lack of wide availability of confirmatory tests further augments this problem. In addition, scrub typhus co-infections with other endemic diseases, due to common behavioural risk factors (i.e. outdoor activity or sleeping, lack of personal protective measures and conducive environment for the vector), increases the need for specific diagnosis [5-9].

In such a scenario, role of rapid diagnostic tests is crucial. With due consideration of the cost, rapidity, single test result and simplicity of interpretation, rapid diagnostic tests have come into vogue. The development of rapid diagnostic tests by the use of immunochromatographic test (ICT) technologies has provided a mechanism for point-of-care serological testing [10]. Use and limitations of immunochromatography based rapid tests have been reported from many countries [11, 12]. In China and Thailand, broadly reactive rapid ICTs for scrub typhus have been evaluated by comparing with standard immunofluorescence $[13,14]$. These ICTs were more sensitive $[13,14]$ and specific [14] than the immunofluorescence assay for the early diagnosis of scrub typhus. The absolute sensitivities of currently useful molecular assays in the acute setting are lower (36-56\%) for the diagnosis of scrub typhus $[15,16]$. These reports suggest the need of sensitive immunoassays to cover the dynamic spectrum of diagnostic positivity.

In addition, cut offs must be validated locally and local strains should be included in the antigen pool for the effective disease diagnosis owing to antigenic diversity in O. tsutsugamushi strains $[10,12]$. Newer tests should not to be validated against IFA alone but instead, be compared against a panel of both serological and antigendetection assays [17]. More emphasis should also be placed on the development of rapid and inexpensive non-serology based means of diagnosis, such as nucleic acid amplification or antigen detection [18].

However, such studies are yet to be reported from India. Recently published DHR-ICMR guidelines for the diagnosis and management of rickettsial diseases in India also discourage the use of rapid tests as they need further evaluation [3]. Hence, diagnostic evaluation of scrub typhus rapid tests in Indian populations is imperative to justify or discourage their use.

\section{Conclusion}

Research studies are needed to find the most suitable test in terms of rapidity of the result, simplicity of the procedure, ease of interpretation and cost to be used in the Indian populace.

\section{Additional file}

Additional file 1: Multilingual abstracts in the six official working languages of the United Nations. (PDF $350 \mathrm{~kb}$ )

Abbreviations

ELISA: Enzyme Linked Immunosorbent assay; PCR: Ploymerase Chain Reaction.

Competing interests

The author declares that there is no conflict of interest or financial disclosure about this publication.

\section{Authors' contribution}

SS searched the literature and drafted the manuscript.

Received: 28 January 2016 Accepted: 21 April 2016

Published online: 12 May 2016

\section{References:}

1. Cowan GO, Friman G, Günther G. Rickettsial infections In Manson's Tropical Diseases. Gordon C (Edi.) 22nd Edi. London Saunders Elsevier Science. Health Sciences Division. 2008:49:894-7.

2. Silpapojakul K. Scrub typhus in the Western Pacific region. Ann Acad Med Singapore. 1997;26:794-800.

3. Rahi M, Gupte MD, Bhargava A, Varghese GM, Arora R. DHR-ICMR Guidelines for diagnosis \& management of Rickettsial diseases in India. Indian J Med Res. 2015;141(4):417-22.

4. Kim DM, Lee YM, Back JH, Yang TY, Lee JH, Song HJ, et al. A serosurvey of Orientia tsutsugamushi from patients with scrub typhus. Clin Microbiol Infect. 2010;16:447-51.

5. Watt G, Jongsakul K, Suttinont C. Possible scrub typhus coinfections in Thai agricultural workers hospitalized with leptospirosis. Am J Trop Med Hyg. 2003;68(1):89-91.

6. Iqbal N, Viswanathan S, Remalayam B, Muthu V, George T. Pancreatitis and MODS Due to Scrub Typhus and Dengue Co-Infection. Trop Med Health. 2012;40(1):19-21.

7. Kumar S, Kumar PS, Kaur G, Bhalla A, Sharma N, Varma S. Rare concurrent infection with scrub typhus, dengue and malaria in a young female. J Vector Borne Dis. 2014:51(1):71-2.

8. Mahajan SK, Kaushik M, Raina R, Thakur P. Scrub typhus and malaria co-infection causing severe sepsis. Trop Doct. 2014;44(1):43-5.

9. Lee CH, Liu JW. Coinfection with leptospirosis and scrub typhus in Taiwanese patients. Am J Trop Med Hyg. 2007;77(3):525-7.

10. Blacksell SD, Jenjaroen K, Phetsouvanh R, Wuthiekanun V, Day NP, Newton PN, Ching WM. Accuracy of AccessBio Immunoglobulin M and Total Antibody Rapid Immunochromatographic Assays for the Diagnosis of Acute Scrub Typhus Infection. Clin Vaccine Immunol. 2010;17(2):263-6.

11. Silpasakorn S, Waywa D, Hoontrakul S, Suttinont C, Losuwanaluk K, Suputtamongkol Y. Performance of SD Bioline Tsutsugamushi assays for the diagnosis of scrub typhus in Thailand. J Med Assoc Thai. 2012;95 Suppl 2:S18-22.

12. Batra HV. Spotted fevers \& typhus fever in Tamil Nadu. Indian J Med Res. 2007:126:101-3.

13. Zhang L, He S, Wang S, Yu H, Li X, Zhang D, et al. Comparison of a rapid diagnostic test and microimmunofluorescence assay for detecting antibody to Orientia tsutsugamushi in scrub typhus patients in China. Asian Pac J Trop Med. 2011;4(8):666-68.

14. Silpasakorn S, Srisamut N, Ekpo P, Zhang Z, Chao CC, Ching WM, et al. Development of new, broadly reactive, rapid IgG and IgM lateral flow assays for diagnosis of scrub typhus. Am J Trop Med Hyg. 2012;87(1):148-52.

15. Zhang S, Song H, Liu Y, Li Q, Wang Y, Wu J, Wan J, et al. Scrub typhus in previously unrecognized areas of endemicity in China. J Clin Microbiol. 2010;48(4):1241-44 
16. Paris DH, Blacksell SD, Nawtaisong P, Jenjaroen K, Teeraratkul A, Chierakul W et al. Diagnostic accuracy of a loop-mediated isothermal PCR assay for detection of Orientia tsutsugamushi during acute Scrub Typhus infection. PLoS Negl Trop Dis. 2011;5(9):e1307.

17. Koh GCKW, Maude RJ, Paris DH, Newton PN, Blacksell SD. Diagnosis of Scrub Typhus. Am J Trop Med Hyg. 2010;82(3):368-70.

18. Blacksell SD, Bryant NJ, Paris DH, Doust JA, Sakoda Y, Day NP. Scrub typhus serologic testing with the indirect immunofluorescence method as a diagnostic gold standard: a lack of consensus leads to a lot of confusion. Clin Infect Dis. 2007:44(3):391-401.

Submit your next manuscript to BioMed Central and we will help you at every step:

- We accept pre-submission inquiries

- Our selector tool helps you to find the most relevant journal

- We provide round the clock customer support

- Convenient online submission

- Thorough peer review

- Inclusion in PubMed and all major indexing services

- Maximum visibility for your research

Submit your manuscript at www.biomedcentral.com/submit
Biomed Central 